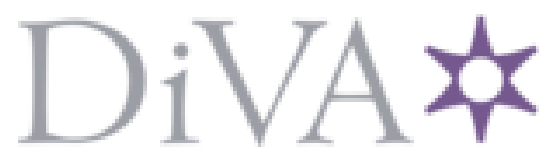

http://www.diva-portal.org

\title{
Postprint
}

This is the accepted version of a paper presented at 2013 IEEE Power \& Energy Society General Meeting.

Citation for the original published paper:

Vardanyan, Y. (2013)

Short-term Hydropower Planning With Uncertain Wind Power Production.

In:

N.B. When citing this work, cite the original published paper.

Permanent link to this version:

http://urn.kb.se/resolve?urn=urn:nbn:se:kth:diva-134309 


\section{Short-term Hydropower Planning With Uncertain Wind Power Production}

\author{
Yelena Vardanyan \\ Electricity Market Research Group \\ KTH Royal Institute of Technology \\ Email: yelena.vardanyan@ee.kth.se
}

\author{
Mikael Amelin \\ KTH Royal Institute of Technology \\ Email: amelin@kth.se
}

\author{
Mohammad Hesamzadeh \\ Electricity Market Research Group \\ KTH Royal Institute of Technology \\ Email: mrhesamzadeh@ee.kth.se
}

\begin{abstract}
The main purpose of this paper is to summarize the findings from simulating two stochastic short-term planning models for a price-taker hydropower producer.

The first model is a two-stage stochastic linear programming problem. Profound sensitivity analysis is provided in terms of volatility in spot market prices and water inflow level. The results show that for the short-term hydropower planning problems the effect of considering price uncertainty in the stochastic model is higher compared to considering inflow level uncertainty.

The second model is a two-stage stochastic linear programming problem. The model generates optimal bids to spot market considering real-time market price uncertainties. While simultaneously bidding to both markets, the results are not realistic. To make the bidding strategies more flexible and robust different approaches are modeled and assessed. Finally one of the approaches is suggested as the most applicable one.
\end{abstract}

\section{INTRODUCTION}

The wind power share in the power system is growing notably [1]. In addition, the wind power prediction techniques are not capable enough to consider wind power production variations, and therefore huge unpredicted wind power production variations might happen in the power system in the near future. These variations must be handled in an optimal way. Some conventional generation units must be used to compensate wind power production variations.

Sweden has a large amount of hydropower, hence, it is highly possible to balance wind power variations with hydropower. Due to the fluctuations in the wind power, the need for balancing power will increase, which will change real-time market prices. Because of its capability to increase production very fast, hydropower with reservoirs can provide balancing power and earn extra profit. Therefore, there is a value of improved planning tools, which allow the hydropower producer to provide even more flexibility.

There are many deterministic models to plan short-term hydropower production in the literature [2], [3], [4] and [5]. However, researchers' interest in work under uncertainty is noticeable recently. Stochastic programming has been used for long-term planning for several decades. With higher levels of non-dispatchable generation, stochastic tools will become necessary also for short-term planning. The following papers apply multi-stage stochastic optimization to plan short-term hydropower production, [6], [7], [8].
Two stochastic models are used in this work. The first model is a two-stage stochastic program under uncertainty related to the spot market prices and water inflow to the reservoirs. The second model is two-stage stochastic model with uncertainty related to spot and real-time market prices.

This paper aims to provide profound sensitivity analysis based on the first model and test different modifications of the second model to provide realistic results. The structure of the paper is the following: section III represents planning models: market prices and hydropower production, section IV states the results, section $\mathrm{V}$ concludes the paper.

\section{Mathematical Modeling}

This section provides background for price modeling in different market places and hydropower production modeling.

\section{A. Modeling of prices in spot and real-time market}

Electricity price forecast is extremely important for all market players and in particular for generating companies, who must manage their units, and the associated economic risk, in a short, medium and long term.

Significant amount of work has been done in the literature related to the modeling and forecasting of the spot market prices. Statistical models like ARIMA and ARMA to forecast spot market prices are applied in [9], [10], [11]. GARCH processes are used to forecast spot market prices in [12]. Moreover, Artificial Neural Networks (ANN) are applied to price forecasting in [13]. In this paper ARIMA technique is used to predict spot market prices.

In contrast with the spot market prices, there are few attempts in the literature to model real-time market prices. An approach to model real time market prices is presented in [14]. The model is built based on the existing dependency between the spot market prices, the traded volumes in the real-time balancing market and the real-time balancing prices. Several approaches to model real-time market prices are presented in [15]. In this work the combination of SARIMA and Markov chain is used to predict real-time market prices.

To build an ARIMA or a SARIMA model, [16], [17] that best fits to the historical time series requires the following steps: Identification, Estimation, Diagnostic checking or validation, Prediction using the constructed model 


\section{B. Short-term hydropower planning}

To have a more realistic plan for the hydropower production, uncertainties have to be considered. For both models it is assumed that the hydropower producer is a price taker. Moreover, the hydropower producer does not have bilateral trading.

The first model is based on the model developed in [6]. However, in addition to uncertainty related to the spot market prices, water inflow level is also considered as random variable. Moreover the bidding rule is simplified. The optimal bidding strategy is a two stage stochastic program. The first stage refers to spot market bidding and the second stage refers to the actual dispatch.

The objective function of the optimization problem applicable for power plants located in a row is presented below.

$$
\mathbf{M} a x \sum_{s, k} P^{s} \pi^{k}\left[\sum_{t}\left(\lambda_{t}^{s} x_{t}^{s, k}+\lambda_{f} \sum_{j} \sum_{r \in R_{j}} \gamma_{r} m_{j}^{T}\right]\right.
$$

The objective function (1) consisting of expected profit from the spot trading and future trading, where $s$ and $k$ are indices for spot price and water inflow scenarios associated with $P^{s}$ and $\pi^{k}$ probabilities respectively. $\lambda_{t}^{s}$ is price in spot market and $\lambda_{f}$ is water opportunity cost. $x_{t}^{s, k}$ is dispatch level to dayahead market, $m_{j, T}^{s, k}$ is the end content of the reservoirs and $\gamma$ is expected future production equivalent for a plant.

$$
x_{t}^{s, k}=\sum_{p=0}^{i} w_{i-p, t} \quad \text { if } \quad \rho_{i} \leq \lambda_{t}^{s} \leq \rho_{i+1},
$$

Constraint (2) states the bidding rule to spot market where $w_{i, t}$ is hourly bid volumes corresponding to the possible bid prices $\rho_{i}$.

$$
\begin{array}{r}
m_{j, t}^{s, k}=m_{j, t-1}^{s, k}-\sum_{n} Q_{j, t, n}^{s, k}-S_{j, t}^{s, k}+\sum_{n} Q_{j-1, t-\tau_{j}, n}^{s, k} \\
+S_{j-1, t-\tau_{j}}^{s, k}+I_{j, t}^{k}, s \in S, t \in T, k \in K,
\end{array}
$$

As it is necessary to keep the hydrological balance between reservoirs a constraint (3) is introduced: for each hour the reservoir's new contents $\left(m_{j, t}^{s, k}\right)$ are equal to its old contents $\left(m_{j, t-1}^{s, k}\right)$ plus water inflow $\left(I_{j, t}^{k}\right)$ minus water outflow. The discharge and the spillage $\left(S_{j-1, t-\tau_{j}}^{s, k}\right)$ from the upper reservoir flow in the reservoir located downstream with delay time $(\tau)$.

$$
G_{j, t}^{s, k} \leq \sum_{n} \mu_{j, n} Q_{j, t, n}^{s, k}
$$

In addition, constraint (4) bounds hourly generation for each operated power plant according to its maximum discharge capacity and marginal production equivalent. Here $G_{j, t}^{s, k}$ is generation level and $Q_{j, t, n}^{s, k}$ is discharged volume for hourly bids.

The quantity traded on the spot market should be equal to the total output of the generation units for each hour. This leads to the constraint (5).

$$
x_{t}^{s, k}=\sum_{j} G_{j, t}^{s, k}, s \in S, t \in T, k \in K,
$$

Last two constraints together put bounds on hourly discharge level and on hourly water storage level respectively.

$$
\begin{gathered}
Q_{j, t, n}^{s, k} \leq \bar{q}_{j, n}, \\
m_{j, t}^{s, k} \leq \bar{m}_{j},
\end{gathered}
$$

Moreover, all variables $\in R_{+}, \bar{q}_{j, n}$ and $\bar{m}_{j}$ are maximum discharge level and maximum reservoir content respectively.

The two-stage stochastic program is summarized below:

\section{Formulation I}

$$
\mathbf{M} a x \sum_{s, k} P^{s} \pi^{k}\left[\sum_{t}\left(\lambda_{t}^{s} x_{t}^{s, k}+\lambda_{f} \sum_{j} \sum_{r \in R_{j}} \gamma_{r} m_{j}^{T}\right]\right.
$$

subject to (2)-(7)

The second model, which is a two-stage stochastic bidding strategy to the spot market while considering the real-time market, is based on the model developed in [15]. The first stage concerns the spot bidding, and the second stage contains electric trading on the real-time market and the actual production planning process. Unlike [15] we used linear bidding rules to real-time market. The main differences of this model and the above explained model are the followings: (1).an extra term in objective function related to the trading on the real-time market (2).limitation on down regulated quantity: the down regulated quantity $d_{s, k, t}^{\downarrow}$ can not exceed the bid volume to spot market for each hour (9).

$$
d_{s, k, t}^{\downarrow} \leq x_{s, t} ;
$$

The complete formulation for the second model follows:

Formulation II

$$
\begin{array}{r}
\mathbf{M} a x \sum_{s} p_{s} \sum_{t} \lambda_{s t} x_{s t}+\sum_{s, l} p_{s} p_{l} \sum_{t} r_{l t}^{\uparrow} d_{s l t}^{\uparrow} \\
-\sum_{s, k} p_{s} p_{k} \sum_{t} r_{k t}^{\downarrow} d_{s k t}^{\downarrow}+\lambda^{\text {future }} \sum_{s, l, k} p_{s} p_{l} p_{k} \sum_{j} \sum_{r \in R_{j}} \gamma_{r} m_{j, T}^{s, l, k}
\end{array}
$$

\section{subject to (2)-(7), (9)}

Note that in (10) $s, l$ and $k$ represent scenarios related to spot, up and down market prices with $p_{s}, p_{l}$ and $p_{k}$ probabilities. $r_{l t}^{\uparrow} / r_{k t}^{\downarrow}$ and $d_{s l t}^{\uparrow} / d_{s k t}^{\downarrow}$ denote the prices and dispatch level to up/down regulating market respectively.

\section{RESUlTS AND DISCUSSION}

For the case study three cascaded reservoirs are studied. The upper reservoir is larger, which is followed by two smaller reservoirs. Every reservoir has local inflow. In addition, each reservoir has a power station which contains a turbine, which by transferring the potential energy of the water to electrical energy generates electricity. The data for the initial reservoirs' content and the inflow level are according to Swedish hydropower plants. Table I summarizes the maximum storage capacity $\bar{m}_{j}$, the maximum discharge $\bar{Q}_{j}$, the maximum production capacity for each power plants $\bar{G}_{j}$ and water delay time $\tau_{j}$. 
Table I

DATA FOR POWER PLANTS

\begin{tabular}{l|l|l|l|l}
\hline & $\bar{m}_{j}(\mathrm{HE})$ & $Q_{j}\left(m^{3} / s\right)$ & $G_{j}(\mathrm{MW})$ & $\tau_{j}(\mathrm{~h})$ \\
\hline I & 305856 & 340 & 95 & 0 \\
\hline II & 1392 & 310 & 50 & 0.5 \\
\hline III & 4008 & 330 & 90 & 2 \\
\hline
\end{tabular}

\section{A. Sensitivity analysis to price and inflow uncertainty}

This study aims to provide profound sensitivity analysis of short-term hydropower planning under uncertainty related to the spot market prices and water inflow to the reservoirs. ARIMA is used to forecast spot market prices and generate scenarios based on that.

Inflow data corresponds to week 16 in 2008. For the spot market price prediction, the data is taken from Nord Pool website from April 20 to June 23, 2010.

The two-stage stochastic programming problem is formulated in GAMS. CPLEX 12.2 solver is used to solve and the execution time is 4.48 seconds. Based on the solution the bidding volume is less than total maximum capacity of the three power plants only in the first, fifth and sixth hours. In the remaining hours it bids with the maximum capacity (235 MWh)

For the comparison purposes and further analysis also the deterministic version of the model is constructed. In the deterministic problem random variables are replaced by their expected values respectively. This is called the expected value (EV) problem [18]. Then, the expected value (EEV) is calculated using the EV solution.

In order to assess the value of knowing and using distributions on future outcomes, the VSS concept is used in stochastic optimization [18]. Therefore, to assess the possible gain from the stochastic model: the effect of including the uncertainties in the model, the VSS quantity is calculated. The VSS is simply the difference of the optimal value of the stochastic problem and the EEV results. Table II demonstrates the calculation result (base case).

Table II

VSS RESULTS FOR DIFFERENT SCENARIOS: THE UNBOLD ROWS EXPRESS THE INITIAL AND THE BOLD ROWS STATES THE SMALL RESERVOIR CASES

\begin{tabular}{l|l|l|l}
\hline & SP & EEV & VSS \\
\hline Base case & 4226871 & 4223197 & 3674 \\
\hline \multirow{2}{*}{ High price vol. } & 4243480 & 4222098 & 21382 \\
& $\mathbf{2 3 3 9 9 7}$ & $\mathbf{2 0 6 1 0 8}$ & $\mathbf{2 7 8 8 9}$ \\
\hline \multirow{2}{*}{ Low price vol. } & 4222352 & 4222301 & 51 \\
& $\mathbf{2 1 3 5 0 2}$ & $\mathbf{2 1 3 3 5 8}$ & $\mathbf{1 4 4}$ \\
\hline \multirow{2}{*}{ High inflow vol. } & 4226709 & 4223040 & 3669 \\
& $\mathbf{2 1 6 8 2 9}$ & $\mathbf{2 0 8 8 2 1}$ & $\mathbf{8 0 0 8}$ \\
\hline \multirow{2}{*}{ Low inflow vol. } & 4226610 & 4222933 & 3677 \\
& $\mathbf{2 1 7 0 3 2}$ & $\mathbf{2 0 8 9 7 5}$ & $\mathbf{8 0 5 7}$ \\
\hline \multirow{2}{*}{ Higher inflow } & 4340100 & 4336491 & 3609 \\
& $\mathbf{3 2 6 2 3 0}$ & $\mathbf{3 0 6 7 8 8}$ & $\mathbf{1 9 4 4 2}$ \\
\hline \multirow{2}{*}{ Lower inflow } & 4169313 & 4165625 & 3688 \\
& $\mathbf{1 5 9 2 0 7}$ & $\mathbf{1 5 5 2 0 1}$ & $\mathbf{4 0 0 6}$ \\
\hline
\end{tabular}

According to the figures in the Table II there is a gain we are using the stochastic rather than deterministic model.
Moreover, to check the sensitivity of the stochastic model towards price volatility and water inflow level volatility, the model is run with the high/low volatile price scenarios and high/low volatile inflow scenarios: for the high volatile cases the standard deviation is taken as double compared with the base case and for the low volatile cases standard deviation is taken as half of the base case. Then VSS values are calculated for each case and are shown in Table II (unbold rows). The comparison in the VSS values shows that noticeably bigger changes happen when different price volatility levels are considered. Small changes in VSS values occur when the models are run with high/low volatile water inflow levels Table II (unbold rows). These results motivate us to investigate further whether it has a big effect to consider water inflow level uncertainty. For that purpose the cases are considered for which the mean of the water inflow level is varied: first the mean is taken twice as big as in the base case, and then the mean is taken half of the mean in the base case. Again the results demonstrate slight changes in the VSS values (last two white rows in Table II.

These comparison results indicates that the effect including price uncertainty in the model is higher compared with the effect of considering water inflow level uncertainty.

To generalize the findings, all these scenarios are studied for a case where three cascaded reservoirs with identical maximum storage capacity is considered. The results have the same profile as the previous case. Again no observable changes in VSS values occur in terms of the cases considered for inflow level. Thus, the conclusion stated in the previous section is not altered.

In order to create a situation where there is an impact of inflow level uncertainty in the model, all those cases are considered where three cascaded small reservoirs are studied. The results are demonstrated in the Table II (grey rows)

From Table II (bold rows) it is possible to see that now there is noticeable change in VSS values for the cases of higher inflow and lower inflow (where mean inflow level is taken double/half as big as in the base case respectively). However, even in this specific structure, which was designed on purpose to see the impact of the inflow level uncertainty, changes in VSS values for inflow level for above mentioned cases are less significant compared with the changes in VSS values in high/low price volatility cases. All these scenarios tend to emphasize the previously drawn conclusion: the impact of including the price uncertainty in the model is higher than that of inflow level uncertainty. In contrast with the spot market prices, within short time period the changes in the inflow level cannot be sharp to have a strong effect.

\section{B. Spot bids considering real-time market: Different ap- proaches to limit real time bids}

The study aims to test the second planning model and comment on the results. According to the results of this model, two extreme cases tend to dominate in the decision making process, which is not realistic. 
This section formulates different approaches which force the optimization model to give more realistic solutions. Each approach is a modification of the base optimization model, that creates bounds on the traded quantity on the real-time market. Every approach is assessed and discussed based on the results.

ARIMA model is used to forecast spot prices and to generate scenarios. Then SARIMA tool together with Markov chain is used to forecast real-time market prices and to generate corresponding price scenarios. Again the GARCH package in MATLAB is used for this purpose. Finally, the optimization model is built in GAMS. The simulated result gives bidding volumes to different market places for each hour, which can be seen from Fig.1. According to Fig.1 for most of the hours

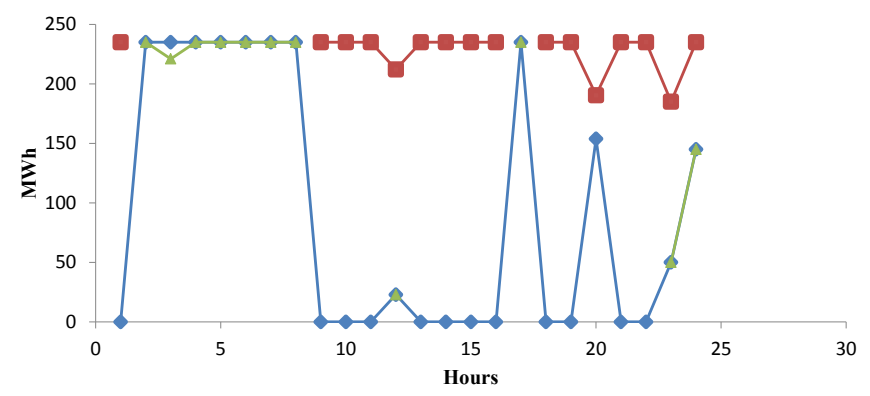

Figure 1. Base case: $\diamond$ represents traded spot volume, $\square / \triangle$ traded up/down regulation volume.

the following happens:

- The maximum amount is bid in upward regulated market;

- The maximum amount is bid to spot market and offered to be bought back in downward market.

The solution is optimal, however the solution is not realistic: in reality the hydropower producer will hardly act in this way. It is expected that the hydropower producer for each hour will bid the main volume to spot market and only small quantity will be offered to the up or down regulating market. This behavior is dependent on the special characteristics of spot and real-time markets. Sales on spot market are definite; when a bid is accepted on spot market, the quantity mentioned on the bid must be provided. In contrast, sales on real-time market are uncertain. Bids submitted to the regulating power market in this work concern as an energy volume in MWh. These bids are activated by the System Operator, when the system needs regulating power. Thus, the amount of regulating power the actor will buy or sell totally depends on the duration the bid has been active within that hour. In addition, the model structure does not consider the next days trading.

The different modifications of the base model are described below.

1) Trivial approach: The first approach is very trivial. It states that the maximum bidden amount to the up/down regulation can not be higher than $20 \%$ of the maximum capacity.

The result, drown in Fig.2, is as naive as the approach itself; whenever it is beneficial to participate in upward/downward regulation market, it bids the allowed amount and the remaining amount is bid to the spot market.

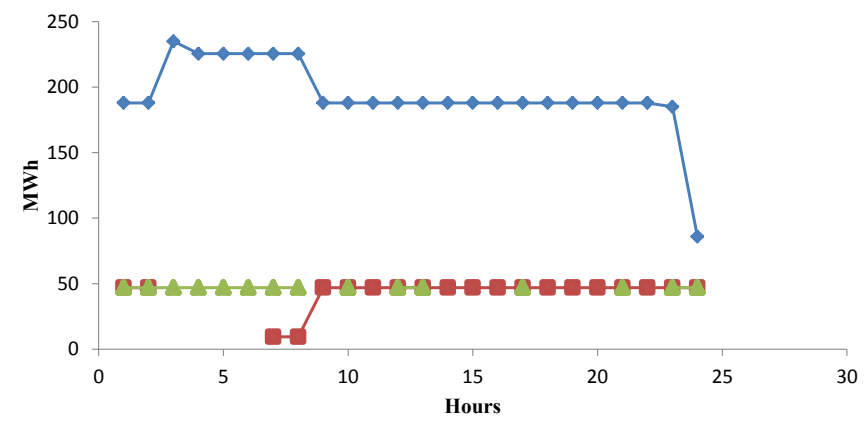

Figure 2. Trivial case: $\diamond$ represents traded spot volume, $\square / \triangle$ traded up/down regulation volume.

2) Limit the end reservoir content approach: The second approach is fixing the reservoirs content at the end of the planning period. The objective function contains penalty cost. Moreover, the constraint should be added to capture the deviation.

Results give more flexible solution, but it is still not very realistic (Fig. 3). In general, the overall results get better, but the improvement is not sharp.

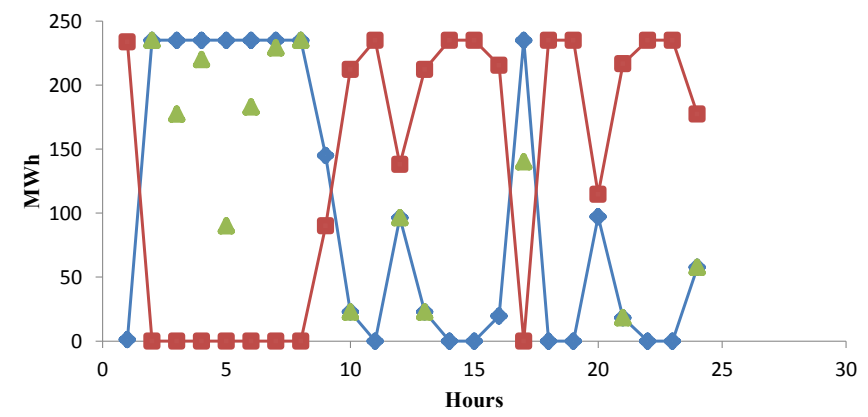

Figure 3. Fixing the end content of reservoirs case: $\diamond$ represents traded spot volume, $\square / \triangle$ traded up/down regulation volume.

3) Non-linear approach: In the third approach we have assumed that real-time market prices are highly influenced by the bidden volume. The real-time market prices are least beneficial, when the bidden volume is equal to the maximum capacity and improving gradually while bidden volume is decreasing.

With the introduction of variable real-time market prices the objective function becomes nonlinear. A local optimum for the optimization problem is also a global optimum if all nonlinear constraints and objective function are convex. However, the objective function is not convex since not all of the eigenvalues are greater than or equal to zero [19]. Thus, we can not claim for the global optimality of the solution. Fig. 4 shows the results. As one can see the results are more realistic in this case: for every hour the base volume is traded in the spot market and only a small amount is bid to upward or downward regulated market. 


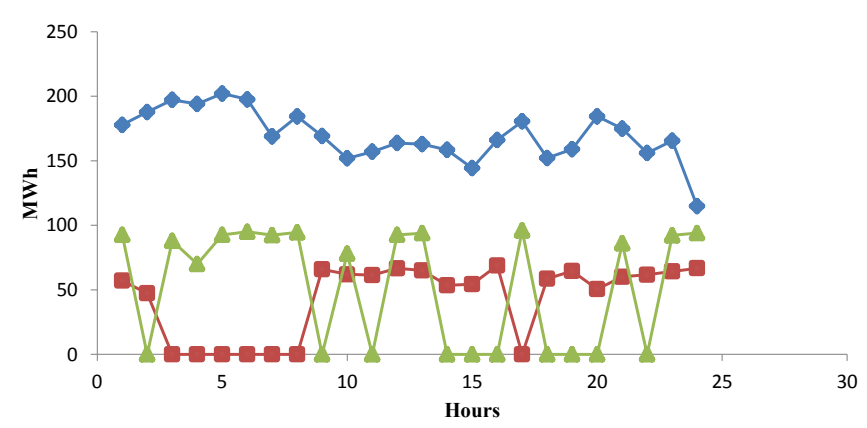

Figure 4. Nonlinear case: $\diamond$ represents traded spot volume, $\square / \triangle$ traded up/down regulation volume.

The main drawback of this approach is that it is not easy to prove the global optimality of the solution. Moreover, it takes 10 hours to solve. Thus, it is clear that the model is not applicable for large systems.

4) Discretized approach: The last approach is the advanced version of the trivial approach. In this case we assume, that available traded amount to upward/downward market has upper bound ( $20 \%$ of the maximum capacity). However, upward/downward market prices are discretized and different percentage of the available amount is bid according to that. The results are drown in the Fig. 5.

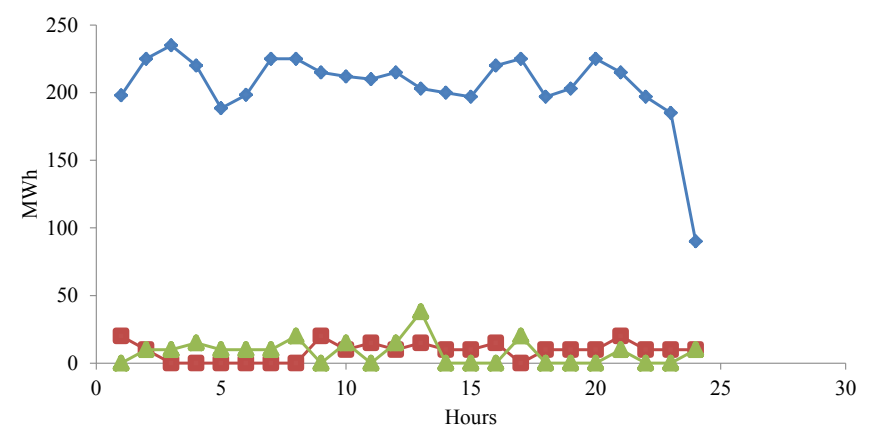

Figure 5. Discretized case: $\diamond$ represents traded spot volume, $\square / \triangle$ traded up/down regulation volume.

In this case also the base quantity is traded in the spot market. Small amount is traded on the upward/downward market. Hence, this approach also gives a realistic solution. The model is linear and solving time is very short.

From all cases above it is obvious that the most promising and applicable approach is the fourth one: it gives flexible results, keeps the optimization problem linear and it is fast to solve.

\section{CONCLUSION}

The continuous growth of wind power creates huge production variation in the electric power system, which has to be handled in an optimal way. Hydropower can compensate these variations, since it is very fast for hydropower plants to change their production.
The first study considered a model, which is an optimal bidding strategy to the spot market. The VSS shows the consequence of considering uncertainties in the problem. Moreover, deep sensitivity analysis has been provided, which shows that for the short-term hydropower planning problems the effect of considering price uncertainty in the stochastic model is higher compared with considering inflow level uncertainty.

The model used in the second study is an optimal bidding strategy to the spot market while considering real-time market. The results of this model are not realistic. Therefore, some modifications of the base model are suggested to provide more robust results. Four different modeling approaches have been tested and evaluated. The fourth approach is suggested as the most promising one.

\section{REFERENCES}

[1] "Global wind 2007 report," tech. rep., Global Wind Energy Council, 2008.

[2] A. Bensalem, A. Miloudi, S. Zouzou, B. Mahdad, and A. Bouhentala, "Optimal short term hydro scheduling of large power systems with descretized horizon," Electrical Engineering, vol. 58, 2007.

[3] A. Borghetti, C. DAmbrosio, A. Lodi, and S. Martello, "An milp approuch for short-term hydro scheduling and unit commitment with head-dependent redservoir," IEEE Transactions on Power Systems, vol. Vol.23, 2008.

[4] D. E. Castronuovo and P. L. J.A., "On the optimization of the dayly operation of a wind-hydro power plan," IEEE Transactions on Power Systems, vol. Vol. 19, 2004.

[5] J. P. S. Catalao, S. J. P. S. Mariano, V. M. F. Mendes, and F. L. A. F. M., "Nonlinear optimization method for short-term hydro scheduling considering head-dependency," IEEE Transactions on Electrical Power, vol. Vol. 20, 2010.

[6] S.-E. Fleten and T. K. Kristoffersen, "Stochastic programming for optimizing bidding strategies of a Nordic hydropower producer," European Journal of Operation Research, vol. Vol. 181, 2007.

[7] S.-E. Fleten and T. K. Kristoffersen, "Short-term hydropower production planning by stochastic programming," Computers and Operations Research, vol. Vol. 35, 2008.

[8] J. G. Gonzalez, R. M. R. Muela, L. M. Santos, and A. M. Gonzalez, "Stochastic joint optimization of wind generation and pumped-storage units in an electricity market," IEEE Transactions on Power Systems, vol. Vol. 23, 2008.

[9] A. J. Conejo, M. A. Plazas, R. Espinola, and A. B. Molina, "Day-ahrad electricity forecasting using the wavelet transform and arima models," IEEE Transactions on Power Systems, vol. Vol. 20, 2005.

[10] J. Contreras, R. Espinola, F. J. Nogales, and A. J. Conejo, "Arima models to predict next-day electricity prices," vol. Vol. 18, no. 3, 2003.

[11] M. Zhou, Z. Yan, Y. X. Ni, G. Li, and Y. Nie, "Electricity price forecasting with confidence-interval estimation through an extended arima approuch," IEEE Proc., vol. Vol. 153, 2006.

[12] R. C. Garcia, J. Contreras, M. van Akkeren, and J. B. C. Garcia, "A garch forecasting model to predict day-ahead electricity prices," vol. Vol. 20, no. 2, 2005.

[13] B. Szkuta, L. Sanabria, and T. Dillon, "Electricity price short-term forecasting using artificial neural networks," 1999.

[14] K. Skytte, "The regulating power market on the Nordic power exchange Nord Pool: an econometric analysis," Energy Economics, vol. Vol. 21, 1999.

[15] M. Olsson, On optimal hydropower bidding in systems with wind power. PhD thesis, Dept. of Electrical Engineering, KTH, 2009.

[16] G. E. P. Box and G. M. Jenkins, Time Series Analysis forecasting and control. HOLDEN-DAY, 1976.

[17] P. J. Brockwell and R. A. Davis, Time Series: Theory and Methods. Springer-Verlag New York Inc., 1991.

[18] J. R. Birge and L. F., Introduction to stochastic programming. SpringerVerlag New York Inc., 1997.

[19] I. Griva, S. G. Nash, and S. A., Linear and Nonlinear Optimization. Sociaty for Industrial and Applied Mathematics, 2009. 